\title{
DETERMINANTES SOCIODEMOGRÁFICOS E PROFISSIONAIS NA CULTURA DE SEGURANÇA DO PACIENTE
}

\section{SOCIO-DEMOGRAPHIC AND PROFESSIONAL DETERMINANTS IN PATIENT SAFETY CULTURE}

\section{DETERMINANTES SOCIODEMOGRÁFICOS Y PROFESIONALES DE LA CULTURA DE SEGURIDAD DEL PACIENTE}

Nylze Helena Guillarducci Rocha ${ }^{1}$, Karoline Faria de Oliveira ${ }^{2}$, Kleiton Gonçalves do Nascimento $^{3}$, Aldenora Laísa Paiva de Carvalho Cordeiro ${ }^{4}$, Vanderlei José Haas ${ }^{5}$, Jacqueline Faria de Oliveira ${ }^{6}$, Maria Helena Barbosa ${ }^{7}$

\section{RESUMO}

Objetivo: avaliar a cultura de segurança do paciente, na ótica dos profissionais de saúde e verificar associação entre a cultura de segurança e características sociodemográficas e profissionais. Métodos: Estudo transversal, quantitativo, cuja amostra foi de 57 profissionais da saúde. Foi utilizado o questionário Safety Attitudes Questionnaire (SAQ), validado para o Brasil. Foram realizadas análises univariadas e análises bivariadas, análise de variância e correlação. Resultados: O escore geral médio do instrumento foi 69,93 pontos. O domínio com melhor escore foi Satisfação no Trabalho $(80,70)$ e com menor escore foi Percepção do Estresse $(64,80)$. Verificaram-se cinco domínios do instrumento com escore médio menor que o considerado ideal pela literatura. Conclusão: Não houve correlação significativa entre as características profissionais e o escore geral ou domínios isolados. A cultura de segurança foi considerada não satisfatória e evidenciou os domínios com maior fragilidade segundo o SAQ: Percepção do Estresse, Percepção da Gerência e Condições de Trabalho.

Descritores: Segurança do Paciente; Gestão da Segurança; Pessoal de Saúde.

\begin{abstract}
Objective: To evaluate patient safety culture from the perspective of health professionals and to assess the association between the safety culture and sociodemographic and professional
\end{abstract}

1 Enfermeira graduada pela Universidade Federal do Triângulo Mineiro-UFTM. Uberaba-MG. E-mail: nylze@hotmail.com

2 Enfermeira do Departamento Didático Científico de Enfermagem na Assistência Hospitalar do Curso de Graduação em Enfermagem da UFTM, Mestre em Atenção à Saúde pela UFTM. Uberaba-MG. E-mail: karol_fmtm2005@yahoo.com.br

3 Enfermeiro do Curso de Graduação em Enfermagem da UFTM, Mestre em Atenção à Saúde pela UFTM. Uberaba-MG. E-mail: Kleiton_uniube@yahoo.com.br

4 Enfermeira do Hospital de Clínicas da UFTM, Mestre em Atenção à Saúde pela UFTM. Uberaba-MG. E-mail: alaisapc@ hotmail.com

5 Físico, Doutor em Física Aplicada à Medicina e Biologia da UFTM. Uberaba-MG. E-mail: vjhaas@uol.com.br 6 Enfermeira do Hospital de Clínicas da UFTM, Mestre em Atenção à Saúde pela UFTM. Uberaba-MG. E-mail: jacquelinefariadeoliveira@gmail.com

7 Enfermeira, Doutora em Enfermagem na Saúde do Adulto, professor associado do Curso de graduação em Enfermagem da UFTM e do Programa de Pós Graduação em Atenção à Saúde da UFTM. Uberaba-MG. E-mail: mhelena331@hotmail.com

*Estudo financiado por Fundação de Amparo à Pesquisa de Minas Gerais (FAPEMIG) 
characteristics. Methods: A cross-sectional, quantitative study of 57 health professionals. The Safety Attitudes Questionnaire (SAQ), validated for Brazil, was used. Univariate and bivariate analysis, analysis of variance and correlation were performed. Results: The mean total score of the instrument was 69.93 points. The domain best score was the Job Satisfaction (80.70) and lowest score was Perceived Stress (64.80). There were five areas of the instrument with a mean score lower than considered ideal for literature. Conclusion: There was no significant correlation between the features professionals and the general score or isolated areas. The safety culture was considered unsatisfactory and showed the areas with greatest weakness Perception of Stress, Perception of Management and Working Conditions, according to the SAQ.

Keywords: Patient Safety; Safety Management; Health Personnel.

\section{RESUMEN}

Objetivo: Evaluar la cultura de seguridad del paciente desde la perspectiva de los profesionales de la salud y evaluar la asociación entre la cultura de la seguridad y las características sociodemográficas y profesionales. Métodos: Estudio transversal, cuantitativo, con una muestra de 57 profesionales de la salud. El cuestionario utilizado actitudes Seguridad Questionnaire (SAQ), validado para Brasil. Se realizaron análisis univariado y bivariado, análisis de varianza y de correlación. Resultados: La media de puntuación total del instrumento fue de 69.93 puntos. La mejor puntuación de dominio fue la satisfacción en el trabajo (80.70) y la más baja puntuación fue el estrés percibido (64.80). Hubo cinco áreas del instrumento con una puntuación media más baja que la considerada ideal para la literatura. Conclusión: No hubo correlación significativa entre las características profesionales y la puntuación en general o en áreas aisladas. La cultura de seguridad se consideró insatisfactoria y mostró las zonas con mayor debilidad El Estrés Percibido, La Percepción de La Administración y Las Condiciones de Trabajo, de acuerdo con la SAQ.

Palabras clave: Seguridad del Paciente; Gestión de la Seguridad; Personal de Salud.

\section{INTRODUÇÃO}

Nos últimos anos, a segurança do paciente tornou-se prioridade na área da saúde e apesar de a assistência à saúde ser algo benéfico para ambas as partes, paciente e profissional, a ocorrência de erros pode trazer danos para os que fazem uso dela. ${ }^{1}$

Adotar uma postura de segurança tornou-se condição indispensável para evitar a ocorrência de eventos adversos (EAs). ${ }^{2}$ Para que ocorra uma mudança efetiva na postura dos profissionais em relação a atitudes seguras, é necessário que os sujeitos desenvolvam conhecimentos e habilidades, que lhes permitam identificar a possibilidade de ocorrência de um erro e saber como agir quando presenciam falhas, que comprometam o bem-estar do paciente. $^{3}$ 
Conhecer o quanto uma instituição de saúde é segura tornou-se um desafio, sendo considerada prioridade para os articuladores políticos, profissionais de saúde e gestores. Torna-se necessário observar alguns aspectos, tanto individuais quanto profissionais, que influenciam negativamente a implementação de um ambiente com ações seguras e atuar sobre eles no planejamento de uma assistência de qualidade. $^{4}$

Além disso, outros fatores nos âmbitos institucional e ambiental podem estar relacionados à adoção de uma cultura de segurança, tais como, estresse profissional, trabalho em equipe, satisfação com o trabalho, estrutura da gerência da instituição e condições de trabalho. ${ }^{5}$

Para que se conheça a realidade de cada instituição hospitalar e se trace um plano de trabalhos voltados à segurança, faz-se necessário identificar a cultura de segurança institucional. Esse conhecimento permite a realização de um diagnóstico situacional em relação a quais fatores, possam influenciar uma postura mais ou menos segura, além da identificação de pontos que poderão ser melhorados.

A identificação dos fatores que se associam ao clima de segurança do paciente é uma importante ferramenta, capaz de diagnosticar fatores que precisam de aprimoramento dentro das instituições de saúde e entre os profissionais, garantindo uma assistência segura e de qualidade aos pacientes. Percebe-se, porém, uma escassez de estudos nacionais realizados com instrumentos de mensuração do clima de segurança de instituições hospitalares. ${ }^{5}$

Surge, portanto, a necessidade de mensurar a cultura de segurança institucional e verificar se esta está relacionada a fatores sociodemográficos e profissionais. Nesse contexto, o objetivo desta pesquisa foi avaliar a cultura de segurança do paciente, na ótica dos profissionais de saúde e verificar a associação entre a cultura de segurança e características sociodemográficas e profissionais.

\section{MATERIAL E MÉTODOS}

Foi realizado um estudo transversal, analítico, com abordagem quantitativa, em um hospital de ensino privado de pequeno porte, que atende pacientes de alta complexidade, localizado no estado de Minas Gerais, Brasil. A instituição possui 50 leitos e 81 profissionais da saúde, que atuam diretamente no atendimento aos pacientes, incluindo enfermeiros, técnicos 
de enfermagem, médicos, nutricionistas, farmacêuticos, psicólogos, fisioterapeutas, assistentes sociais, técnicos em farmácia e técnicos em radiologia. Como critérios de inclusão, foram considerados: estar lotado em suas unidades de atuação há mais de 1 mês e possuir regime de trabalho mínimo de 20 horas semanais.

A população alvo $(\mathrm{N})$ foi de 81 profissionais, e a população de acesso (n) que participou do estudo foi de 57. A alocação dos participantes foi por amostra

de conveniência, conforme a aceitação dos profissionais em participar da pesquisa.

Para o cálculo amostral, foi considerado um coeficiente de correlação de Pearson positivo, $r=0,4$, entre o tempo de formação e o escore total de segurança do paciente, para um nível de significância de 0,05 e um erro tipo II de 0,1, resultando em um poder apriorístico de $90 \%$. Empregando-se o aplicativo PASS 2002, chega-se a um tamanho amostral de 61. Contudo, devido às perdas, o número final de participantes foi de 57.

O diagrama a seguir representa as perdas e exclusões da pesquisa (Figura 1):

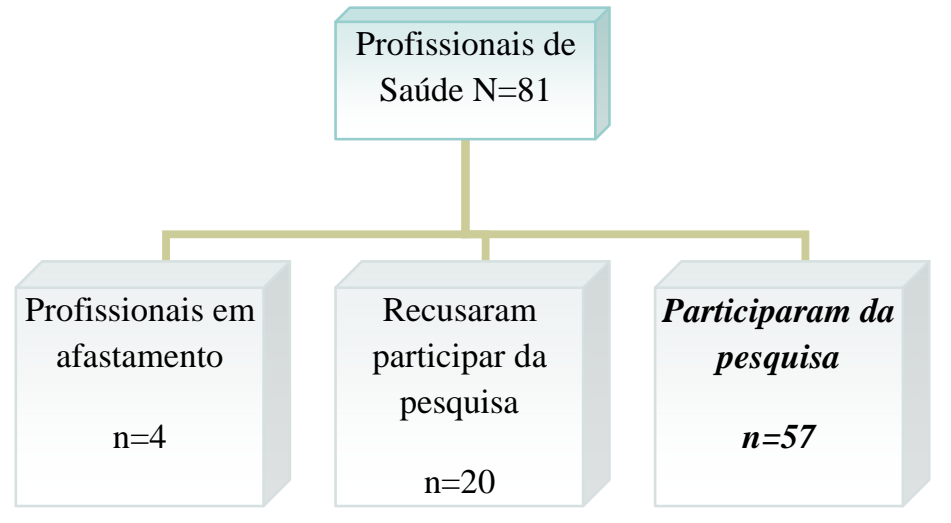

Figura 1: Fluxograma da amostra.

Para obtenção dos dados, utilizou-se o instrumento denominado Safety Attitudes Questionnaire (SAQ), validado para o idioma português (Brasil). ${ }^{6} \mathrm{O}$ questionário contém 41 itens, que correspondem a seis domínios: 1. Clima de Trabalho em Equipe (a qualidade do relacionamento e a colaboração entre os membros de uma equipe); 2. Clima de Segurança (percepção dos profissionais quanto ao comprometimento organizacional para segurança do paciente); 3. Satisfação no Trabalho (visão positiva do local de trabalho); 4. Percepção do Estresse 
(reconhecimento de quanto os fatores estressores podem influenciar na execução do trabalho); 5. Percepção da Gerência (aprovação das ações da gerência ou administração na unidade em que o profissional atua ou no hospital) e 6 . Condições de Trabalho (qualidade do ambiente de trabalho). ${ }^{1}$

A resposta de cada item pode ser na escala de cinco pontos de Likert ("discorda totalmente", “discorda parcialmente", "neutro", "concorda parcialmente", "concorda totalmente") ou "não se aplica". O escore final da escala varia de 0 a 100 , onde zero é a pior percepção do clima de segurança e 100, a melhor percepção. Segundo os autores originais do instrumento, são considerados escores positivos as pontuações maiores ou iguais a $75 .{ }^{7}$

Para assegurar o anonimato dos participantes, os Termos de Consentimento Livre e Esclarecido (TCLE) foram entregues para assinatura e, após o consentimento, foram guardados separadamente do instrumento de coleta de dados (SAQ), em envelope próprio. Para a obtenção dos dados, o SAQ foi entregue aos participantes, após a assinatura do TCLE, para que fosse preenchido após seu turno de trabalho, sem interferir em suas atividades profissionais. Foi determinado o prazo de 24 horas para a devolução aos pesquisadores do instrumento preenchido.

Para a caracterização da amostra utilizou-se um formulário que abordava os aspectos sociodemográficos e profissionais, obtidos no momento da entrega do SAQ preenchido pelo profissional.

Os dados da pesquisa foram validados por dupla digitação em uma planilha eletrônica do programa Excel® para Windows®. Posteriormente, o processamento e a análise das informações foram realizados no programa Statistical Package for the Social Sciences (SPSS) versão 19.0 para Windows®. Para o cálculo dos escores do SAQ, por domínio, somaram-se as respostas dos itens de cada domínio e dividiu-se o resultado pelo número de itens correspondente a cada domínio, com base na fórmula $(m-1) \times 25$, onde $m$ é a média dos itens do domínio em questão, variando no intervalo [0-100].

Para avaliar a influência das variáveis categóricas sobre os escores de segurança, utilizou-se o teste t de Student (categóricas dicotômicas) e, para as variáveis ordinais, o teste de correlação de Spearman. As análises foram consideradas 
estatisticamente significativas quando $\mathrm{p} \leq 0,05$.

Este estudo foi aprovado pelo Comitê de Ética em Pesquisa com Seres Humanos da Universidade Federal do Triângulo Mineiro (CEP-UFTM), Parecer número 2306, em conformidade com a Resolução $n^{\circ}$ 196/96 do Ministério da Saúde sobre pesquisa envolvendo seres humanos. Teve apoio financeiro da Fundação de Amparo à Pesquisa do Estado de Minas Gerais (FAPEMIG).

\section{RESULTADOS}

\section{Aspectos sociodemográficos e}

\section{profissionais da equipe de saúde}

Verificou-se que a maioria, 42 $(73,7 \%)$, dos profissionais de saúde participantes do estudo era do sexo feminino; e $36(63,2 \%)$ atuavam apenas com pacientes adultos. A maioria dos profissionais compunha a equipe de enfermagem, sendo 33 técnicos de enfermagem $(57,89 \%)$ e, 9 enfermeiros $(15,79 \%)$.

Dos outros profissionais, $6(10,53 \%)$ eram técnicos de farmácia, $2(3,51 \%)$ eram médicos; e $2(3,51 \%)$ eram nutricionistas. Dentre os outros profissionais, participaram da pesquisa um psiquiatra, um técnico em radiologia, um farmacêutico, um fisioterapeuta e um assistente social. A maioria dos profissionais, $37(64,9 \%)$, exercia apenas assistência direta aos pacientes.

Em relação à formação profissional, $20(35,1 \%)$ haviam-na concluído entre 5 a 10 anos antes da pesquisa. A maioria dos participantes não possuía outro vínculo empregatício, 41 (71,9\%), e 44 (77,2\%) não possuíam pós-graduação.

Quanto ao setor de atuação, 27 $(47,4 \%)$ atuavam em área semicrítica e 17 $(29,8 \%)$ em áreas críticas. Observou-se que a maioria dos profissionais, 14 (24,6\%), tinham entre 3 e 4 anos de atuação profissional. Houve prevalência de 1 a 2 anos de vínculo com a instituição onde a pesquisa foi realizada, $14(24,6 \%)$.

A tabela 1, a seguir, apresenta a caracterização sociodemográfica e profissional dos participantes da pesquisa. 
Tabela 1. Características sociodemográficas e profissionais dos participantes da pesquisa. Minas Gerais, Brasil, 2014.

\begin{tabular}{|c|c|c|c|}
\hline Variáveis & & $\mathbf{n}$ & $\%$ \\
\hline \multirow[t]{2}{*}{ Sexo } & Masculino & 15 & 26,3 \\
\hline & Feminino & 42 & 73,7 \\
\hline \multirow[t]{10}{*}{ Profissão } & Técnico de Enfermagem & 33 & 57,8 \\
\hline & Enfermeiro & 9 & 15,7 \\
\hline & Técnico em Farmácia & 6 & 10,5 \\
\hline & Médico & 2 & 3,5 \\
\hline & Nutricionista & 2 & 3,5 \\
\hline & Técnico em Radiologia & 1 & 1,8 \\
\hline & Psicólogo & 1 & 1,8 \\
\hline & Farmacêutico & 1 & 1,8 \\
\hline & Fisioterapeuta & 1 & 1,8 \\
\hline & Assistente Social & 1 & 1,8 \\
\hline \multirow[t]{3}{*}{ Atuação Principal } & Adulto & 36 & 63,2 \\
\hline & Pediátrico & 5 & 8,8 \\
\hline & Ambos & 16 & 28,1 \\
\hline \multirow[t]{3}{*}{ Atuação Profissional } & Somente Assistencial & 37 & 64,9 \\
\hline & Somente Administrativo & 3 & 5,3 \\
\hline & Ambos & 17 & 29,8 \\
\hline \multirow[t]{11}{*}{ Setor de Atuação } & Bloco Cirúrgico & 5 & 8,8 \\
\hline & Pronto Socorro Adulto & 1 & 1,8 \\
\hline & UTI Adulto & 11 & 19,3 \\
\hline & Clínica Cirúrgica & 2 & 3,5 \\
\hline & Clínica Médica & 13 & 22,8 \\
\hline & Berçário & 2 & 3,5 \\
\hline & Pediatria & 1 & 1,8 \\
\hline & Ginecologia e Obstetrícia & 9 & 15,8 \\
\hline & Serviço de Som e Imagem & 1 & 1,8 \\
\hline & Nutrologia & 4 & 7,0 \\
\hline & Comissão de Controle de Infecção & 1 & 1,8 \\
\hline \multirow[t]{7}{*}{ Tempo na Especialidade } & Menos de 6 meses & 7 & 12,3 \\
\hline & 6 a 11 meses & 9 & 15,8 \\
\hline & 1 a 2 anos & 13 & 22,8 \\
\hline & 3 a 4 anos & 14 & 24,6 \\
\hline & 5 a 10 anos & 9 & 15,8 \\
\hline & 11 a 20 anos & 2 & 3,5 \\
\hline & 21 anos ou mais & 3 & 5,3 \\
\hline \multirow{6}{*}{$\begin{array}{l}\text { Tempo em que Atua } \\
\text { na Instituição }\end{array}$} & Menos de 6 meses & 13 & 22,8 \\
\hline & 6 a 11 meses & 10 & 17,5 \\
\hline & 1 a 2 anos & 14 & 24,6 \\
\hline & 3 a 4 anos & 5 & 8,8 \\
\hline & 5 a 10 anos & 12 & 21,1 \\
\hline & 11 a 20 anos & 3 & 5,3 \\
\hline \multirow[t]{5}{*}{ Tempo de Formação } & 6 a 11 meses & 2 & 3,5 \\
\hline & 1 a 2 anos & 11 & 19,3 \\
\hline & 3 a 4 anos & 15 & 26,3 \\
\hline & 5 a 10 anos & 20 & 35,1 \\
\hline & 11 a 20 anos & 6 & 10,5 \\
\hline
\end{tabular}




\begin{tabular}{clccc}
\hline & 21 anos ou mais & 3 & 5,3 \\
Pós-Graduação & Sim & 13 & 22,8 \\
& Não & 44 & 77,2 \\
Tipo de Pós-Graduação & Latu Sensu & 13 & 22,8 \\
& Stricto Sensu & - & - \\
\multirow{2}{*}{ Outro Vínculo Empregatício } & Não se aplica & Sim & 44 & 77,2 \\
& Não & 16 & 28,1 \\
& & 41 & 71,9 \\
\hline
\end{tabular}

\section{Análise do Safety Attitudes}

\section{Questionnaire(SAQ)}

O escore geral médio obtido pelo instrumento foi $69,93 \quad(\mathrm{~S}=15,64)$, com mínimo de 18,13 e máximo de 92,68 pontos.

Com base nos escores por domínios, nota-se que o terceiro domínio, referente à satisfação no trabalho, demonstrou o maior escore, com média de 80,70 ( $\mathrm{S}=20,09)$, o que representa uma percepção positiva em relação a questões que envolvem o seu trabalho.
O domínio 4, que avalia a percepção do estresse, apresentou o menor escore, obtendo uma média de 64,80 pontos ( $S=24,30)$, um resultado desfavorável, pois evidencia que os profissionais não percebem o quanto fatores estressores podem influenciar na execução do trabalho.

A Tabela 2 apresenta as análises descritivas dos escores em relação aos domínios.

Tabela 2. Distribuição da análise dos escores por domínios. Minas Gerais, Brasil, 2014.

\begin{tabular}{ccccccc}
\hline Estatística & $\begin{array}{c}\text { Clima de } \\
\text { Trabalho } \\
\text { em Equipe }\end{array}$ & $\begin{array}{c}\text { Clima de } \\
\text { Segurança }\end{array}$ & $\begin{array}{c}\text { Satisfação } \\
\text { no } \\
\text { Trabalho }\end{array}$ & $\begin{array}{c}\text { Percepção } \\
\text { do } \\
\text { Estresse }\end{array}$ & $\begin{array}{c}\text { Percepção } \\
\text { da } \\
\text { Gerência }\end{array}$ & $\begin{array}{c}\text { Condições } \\
\text { de } \\
\text { Trabalho }\end{array}$ \\
\hline Média & 72,62 & 71,91 & 80,70 & 64,80 & 65,02 & 67,32 \\
Desvio Padrão & 19,78 & 16,48 & 20,09 & 24,30 & 23,38 & 29,28 \\
Mínima & 20,83 & 28,57 &, 00 & 18,75 & 9,09 &, 00 \\
Máxima & 100,00 & 100,00 & 100,00 & 100,00 & 100,00 & 100,00 \\
\hline
\end{tabular}


A seguir, a tabela 3 mostra a frequência de respostas dos itens 14, 33, 34, 35 e 36, considerados itens isolados, segundo as normas do instrumento SAQ.

Nota-se que a maioria das respostas foram "concordo totalmente".

Tabela 3. Frequência de respostas dos participantes para itens que não correspondem a nenhum domínio. Minas Gerais, Brasil, 2014.

\begin{tabular}{|c|c|c|c|c|c|c|}
\hline $\begin{array}{c}\text { Itens isolados do SAQ } \\
\mathrm{n}(\%)\end{array}$ & 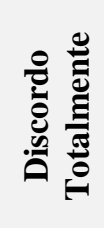 & 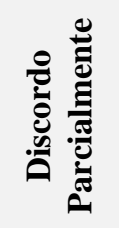 & 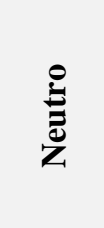 & 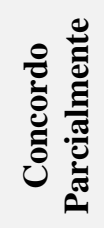 & 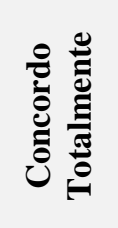 & 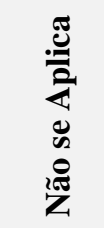 \\
\hline $\begin{array}{l}\text { 14. Minhas sugestões sobre segurança } \\
\text { seriam postas em ação se eu as } \\
\text { expressasse à administração. }\end{array}$ & $\begin{array}{c}9 \\
(15,8)\end{array}$ & $\begin{array}{c}3 \\
(5,4)\end{array}$ & $\begin{array}{c}19 \\
(33,3)\end{array}$ & $\begin{array}{c}10 \\
(17,5)\end{array}$ & $\begin{array}{c}10 \\
(17,5)\end{array}$ & $\begin{array}{c}6 \\
(10,5)\end{array}$ \\
\hline $\begin{array}{l}\text { 33. Eu vivencio boa colaboração com } \\
\text { os(as) enfermeiros(as)nesta área }\end{array}$ & $\begin{array}{c}1 \\
(1,7)\end{array}$ & $\begin{array}{c}1 \\
(1,7)\end{array}$ & $\begin{array}{c}10 \\
(17,5)\end{array}$ & $\begin{array}{c}13 \\
(22,9)\end{array}$ & $\begin{array}{c}31 \\
(54,5)\end{array}$ & $\begin{array}{c}1 \\
(1,7)\end{array}$ \\
\hline $\begin{array}{l}\text { 34. Eu vivencio boa colaboração com a } \\
\text { equipe de médicos nesta área. }\end{array}$ & $\begin{array}{c}2 \\
(3,5)\end{array}$ & $\begin{array}{c}4 \\
(7,0)\end{array}$ & $\begin{array}{c}7 \\
(12,3)\end{array}$ & $\begin{array}{c}18 \\
(31,6)\end{array}$ & $\begin{array}{c}26 \\
(45,6)\end{array}$ & - \\
\hline $\begin{array}{l}\text { 35. Eu vivencio boa colaboração com } \\
\text { os farmacêuticos nesta área. }\end{array}$ & $\begin{array}{c}2 \\
(3,5)\end{array}$ & $\begin{array}{c}2 \\
(3,5)\end{array}$ & $\begin{array}{c}9 \\
(15,8)\end{array}$ & $\begin{array}{c}14 \\
(24,6)\end{array}$ & $\begin{array}{c}29 \\
(50,9)\end{array}$ & $\begin{array}{c}1 \\
(1,7)\end{array}$ \\
\hline $\begin{array}{l}\text { 36. Falhas na comunicação que levam } \\
\text { a atrasos no atendimento são comuns. }\end{array}$ & $\begin{array}{c}8 \\
(14,0) \\
\end{array}$ & $\begin{array}{c}8 \\
(14,0) \\
\end{array}$ & $\begin{array}{c}12 \\
(21,1) \\
\end{array}$ & $\begin{array}{c}16 \\
(28,1) \\
\end{array}$ & $\begin{array}{c}12 \\
(21,1) \\
\end{array}$ & $\begin{array}{c}1 \\
(1,7) \\
\end{array}$ \\
\hline
\end{tabular}

Em relação à análise bivariada, não houve diferença entre os sexos $(p>0,05)$; ou com a realização de pós-graduação (Tabela 4). Não houve correlação entre o tempo na especialidade, o tempo de formação ou o tempo em que atua na instituição e o escore geral ou de domínios isolados $(\mathrm{p}>0,05)$.

Tabela 4. Distribuição da diferença de média entre sexo, realização de pós-graduação, presença de outro vínculo empregatício e atuação profissional em relação à pontuação geral e por domínios. Minas Gerais, Brasil, 2014.

\begin{tabular}{cccccccc}
\hline Variável & $\begin{array}{c}\text { Pontuação } \\
\text { Geral }\end{array}$ & $\begin{array}{c}\text { Clima de } \\
\text { Trabalho } \\
\text { em Equipe }\end{array}$ & $\begin{array}{c}\text { Clima de } \\
\text { Segurança }\end{array}$ & $\begin{array}{c}\text { Satisfação } \\
\text { no } \\
\text { Trabalho }\end{array}$ & $\begin{array}{c}\text { Percepção } \\
\text { do Estresse }\end{array}$ & $\begin{array}{c}\text { Percepção } \\
\text { da } \\
\text { Gerência }\end{array}$ & $\begin{array}{c}\text { Condições } \\
\text { de } \\
\text { Trabalho }\end{array}$ \\
\hline $\begin{array}{c}\text { Masculino } \\
\text { Média / (DP*) }\end{array}$ & 55,87 & 73,19 & 71,38 & 76,66 & 64,86 & 61,85 & 61,67 \\
& $(20,68)$ & $(19,55)$ & $(21,68)$ & $(25,75)$ & $(18,38)$ & $(26,09)$ & $(21,42)$ \\
Feminino & 70,83 & 72,42 & 75,35 & 82,14 & 64,78 & 66,15 & 69,35 \\
Média / (DP*) & $(14,61)$ & $(20,09)$ & $(20,85)$ & $(17,81)$ & $(16,29)$ & $(22,55)$ & $(28,60)$ \\
$\mathbf{p}^{\dagger}$ & 0,28 & 0,89 & 0,35 & 0,46 & 0,99 & 0,57 & 0,41
\end{tabular}




\begin{tabular}{cccccccc} 
Pós Graduado & 76,40 & 74,03 & 84,07 & 84,23 & 73,71 & 73,60 & 72,77 \\
Média / (DP*) & $(13,46)$ & $(22,70)$ & $(11,40)$ & $(14,41)$ & $(25,72)$ & $(18,91)$ & $(20,80)$ \\
& & & & & & & \\
Graduado & 64,08 & 72,21 & 83,21 & 79,66 & 62,16 & 62,48 & 65,81 \\
Média / (DP*) & $(32,32)$ & $(19,11)$ & $(10,41)$ & $(21,52)$ & $(23,53)$ & $(24,14)$ & $(21,39)$ \\
& 0,06 & 0,79 & 0,22 & 0,38 & 0,16 & 0,09 & 0,38 \\
\hline $\mathbf{p}^{\dagger}$ & &
\end{tabular}

"DP: Desvio Padrão; ${ }^{\dagger}$ p: valor de p (teste T Student), significativo se p<0,05; ${ }^{\ddagger}$ : valor de p (teste Anova)

\section{DISCUSSÃO}

Dentre os profissionais da equipe multiprofissional de saúde, participantes do estudo, a equipe de enfermagem foi a mais prevalente. Esse fato também foi observado em estudo de validação das propriedades psicométricas do instrumento SAQ na língua alemã, realizado em dois hospitais universitários da Suíça $^{8}$ e em estudo realizado na rede de hospitais públicos da região de Murcia, na Espanha. ${ }^{9}$

Isso pode ser visto como ponto positivo, pois são profissionais que estão envolvidos em período contínuo com o paciente, sendo atribuição dos enfermeiros a prática assistencial e gerencial, e dos técnicos de enfermagem a prestação de cuidado contínuo aos pacientes. ${ }^{10}$

Observou-se uma alta rotatividade dos profissionais atuantes na instituição e predomínio de não envolvimento dos profissionais com pós-graduações. A consequência da alta rotatividade é sentida no declínio da qualidade, por levar à contratação de novos membros para a equipe, que não conhecem a rotina do serviço. ${ }^{11}$ Resultados semelhantes foram encontrados em pesquisa utilizando o SAQ, realizada em hospital especializado em oncologia de Minas Gerais, Brasil. Qualificações e especializações atualizam o conhecimento, que é dinâmico na área da saúde, com o uso de novas tecnologias ou evidências novas em relação a tratamentos e terapias. ${ }^{12}$

A maioria dos profissionais não possuía outro vínculo empregatício, o que corrobora estudo que avaliou a cultura de segurança do paciente utilizando o SAQ, ${ }^{12}$ e representa uma situação favorável. Jornadas extras demandam cargas excessivas de trabalho, que podem gerar cansaço físico e mental, além de estresse para o profissional, e assim desencadear problemas relacionados à segurança do paciente. A exaustão física e mental tem influência no aumento de taxa de absenteísmo,e na rotatividade nos serviços e leva a uma redução das atividades 
cognitivas por parte do funcionário, o que desencadeia um prejuízo da qualidade da assistência. $^{12-13}$

Em relação aos domínios avaliados, o domínio Percepção do Trabalho em Equipe obteve uma média inferior a 75 pontos, similar a estudo que teve como objetivo explorar a variabilidade nas dimensões de cultura de segurança entre áreas clínicas da Suíça e dos Estados Unidos. ${ }^{14}$ Dessa forma, pode-se observar que os profissionais possuem percepções negativas a cerca do Clima de Trabalho em Equipe. Esse fato pode se refletir em problemas interpessoais constantes, equipe não cooperativa e trabalhadores com sentimento de opiniões desprezadas sobre assuntos do cotidiano. ${ }^{14}$ Nos hospitais onde ocorre um bom clima de trabalho em equipe, os índices de satisfação do paciente, qualidade e segurança do cuidado e resultados da equipe de enfermagem são melhores. $^{15}$

O desenvolvimento de estratégias para melhorar o ambiente de trabalho em equipe pode significar um investimento de baixo custo. ${ }^{15}$ Ações como o estabelecimento de programas de formação de equipe interdisciplinar, a construção e manutenção de programas de transferência de informação; o treinamento de técnicas de resolução de problemas e o uso da educação permanente podem ser estratégias importantes para esse fim. ${ }^{16}$

O domínio Clima de Segurança obteve pontuação semelhante aos estudos que utilizaram o SAQ, realizados nos Estados Unidos ${ }^{14}$ e na Índia. ${ }^{17}$ Dessa forma, nota-se que o clima de segurança neste hospital está deficiente em relação ao recomendado pelos autores do instrumento. Esta percepção está relacionada ao comprometimento organizacional para segurança do paciente.

Para determinação de um ambiente com clima seguro é necessário que equipe e gestores trabalhem juntos a fim de proporcionar ao cliente um cuidado de qualidade. Medidas como implantação de política de relato dos erros sem penalização, reformulação dos processos administrativos, que permita aos trabalhadores se sentirem confortáveis em discutir suas preocupações sobre segurança e a criação de grupos de discussão de erros contribuem para a melhoria e a manutenção do clima de segurança. ${ }^{18}$

De acordo com os resultados deste estudo, o domínio Satisfação no Trabalho evidenciou a maior média, com 80,70 pontos, semelhante a estudo que avaliou a percepção do clima de segurança na 
percepção de profissionais de enfermagem ${ }^{1}$ e a estudo realizado em centro de oncologia. $^{12}$ Esse domínio avalia a prestação de cuidados ao paciente com segurança e qualidade e o alto grau de comprometimento e performance na assistência prestada. ${ }^{1,13}$ Possivelmente, isso indica que, apesar do clima de segurança geral encontrado no estudo apresentar baixa pontuação, os profissionais se consideram satisfeitos em relação a sua prestação de cuidados e ao seu desempenho em relação à própria função.

A questão de Percepção do Estresse, tratada no domínio 4 relacionado com os fatores extrínsecos que interferem na qualidade do trabalho, apresentou uma média de 64,80 pontos, similar a estudo brasileiro $^{12}$ e a estudo norte-americano. ${ }^{14}$

Esse domínio reflete o reconhecimento dos profissionais de que situações, como carga de trabalho excessiva e cansaço são desencadeadoras de problemas no atendimento aos pacientes, oferecendo riscos para estes. Quanto mais alta a pressão no ambiente de trabalho, maiores serão as chances para ocorrência de eventos adversos ou mesmo de acidentes de trabalho que comprometem a qualidade da assistência prestada. ${ }^{19}$
O domínio que avalia a Percepção da Gerência da Unidade e do Hospital, com média de 65,02 pontos, corrobora o encontrado em outros estudos. ${ }^{1,12,14}$ Essa média é inferior ao recomendado pela literatura para uma boa percepção do domínio. Pode refletir que os profissionais consideram a administração não preocupada com o bem estar dos pacientes e deles mesmos. ${ }^{14}$

É importante que o profissional esteja envolvido com as ações tomadas pelas administrações do hospital e seus setores de trabalho, a fim de proporcionar ao paciente cuidados seguros. ${ }^{1}$ A criação de uma atmosfera no ambiente de trabalho propícia a um diálogo aberto sobre erros, a manutenção de um ambiente não punitivo e o treinamento contínuo dos profissionais são algumas das principais ações da administração do hospital e da unidade que podem causar impacto positivo na segurança do paciente. ${ }^{16}$

Em relação à percepção das condições de trabalho, abordada no domínio 6, a média do escore obtida foi de 67,32 , semelhante a outros estudos ${ }^{1,12,14}$ e inferior ao escore 75 considerado como limite para uma boa percepção do domínio. Isso pode significar deficiências nos programas de treinamentos, participação 
não representativa pelos profissionais e na solução de problemas e fornecimento inadequado de informações para os pacientes, indicando a necessidade de ações de melhoria. ${ }^{19}$

Apesar de não haver correlação estatisticamente significativa entre as variáveis sociodemográficas e profissionais, considera-se importante destacar o elevado número de profissionais que não realizaram qualificações posteriores à formação profissional básica.

A cada dia percebe-se um aumento da exigência pelo mercado de trabalho de qualificação e especialização profissional, que tragam um diferencial e valorizem os profissionais. A busca de capacitações atualiza o conhecimento, que é dinâmico na área da saúde, com o uso de novas tecnologias ou evidências novas em relação a tratamentos e terapias. ${ }^{12}$ Além disso, a capacitação profissional é considerada uma necessidade para desmistificar alguns paradigmas que impedem a mudança da cultura de alguns profissionais da saúde ${ }^{20}$.

\section{CONCLUSÃO}

A avaliação da percepção dos profissionais da equipe de saúde em relação à cultura de segurança foi negativa de forma geral, ou seja, apresentou pontuação inferior ao recomendado como ideal pela literatura. A pontuação do clima de segurança institucional foi comprometida principalmente devido às pontuações referentes à percepção da gerência da unidade e do hospital, à percepção do estresse e às condições de trabalho.

Ao associar as características sociodemográficas e profissionais, não se verificou associação estatisticamente significativa do clima de segurança com o sexo (feminino ou masculino) ou o nível de escolaridade (possuir ou não pósgraduação) em relação ao escore geral ou em cada domínio. Não foi encontrada correlação estatisticamente significativa entre os escores e o tempo de formação, o tempo de atuação na instituição ou o tempo na especialidade.

Como limitação deste estudo, embora tenha sido utilizada amostragem aleatória simples para obtenção do tamanho amostral, o número de profissionais da saúde que compunha a população estudada era próximo ao número de profissionais necessário para compor a amostra. Como o número de recusas foi de quase um quarto da população, a obtenção dos dados pode 
limitar o espectro da análise. Contudo, os objetivos propostos foram atingidos. Sugere-se que estudos longitudinais sejam realizados futuramente.

\section{REFERÊNCIAS}

1. Rigobello MCG, Carvalho REFL, Cassiani SHB, Galon T, Capucho HC, Deus NN. The climate of patient safety: perception of nursing professionals. Acta Paul Enferm [Internet]. 2012 [access: 12 nov 2015]; 25(5): 728-735. Available from: http://dx.doi.org/10.1590/S010321002012000500013.

2. Tobias GC, Bezerra ALQ, Branquinho NCS, Camargo Silva AEB. Cultura de la seguridad del paciente en la atención sanitaria: un estudio bibliométrico. Enferm Glob [revista en Internet]. 2014 [acceso: 5 oct 2015]; 13(33): 336-348. Disponible en: http://dx.doi.org/10.6018/eglobal.13.1.159 931.

3. Yoshikawa JM, Sousa BEC, Peterlini MAS, Kusahara DM, Pedreira MLG, Avelar AFM. Comprehension of undergraduate students in nursing and medicine on patient safety. Acta Paul Enferm [Internet]. 2013 [access: 5 may 2016], 26(1):21-29. Avaliable from: http://dx.doi.org/10.1590/S010321002013000100005.

4. El-Jardali F, Sheikh F, Garcia NA, Jamal D, Abdo A. Patient safety culture in a large teaching hospital in Riyadh: baseline assessment, comparative analysis and opportunities for improvement. BMC Health Services Research [Internet]. 2014 [access: 20 nov 2016];14(122):1-15. Available from: doi: 10.1186/1472-696314-122.

5. Luiz RB, Simões ALA, Barichello, E, Barbosa MH. Factors associated with the patient safety climate at a teaching hospital. Rev. Latino-Am. Enfermagem [Internet]. 2015 [access: 12 apr 2016], 23(5): 880-887. Available from: http://dx.doi.org/10.1590/01041169.0059.2627.

6. Carvalho REFL, Cassiani SHB. Crosscultural adaptation of the Safety Attitudes Questionnaire - Short Form 2006 for Brazil. Rev. Latino-Am. Enfermagem [Internet]. 2012 [access: 12 apr 2016]; 20(3): [8 telas]. Available from: http://www.revistas.usp.br/rlae/article/view /48581/52545.

7. Sexton JB, Thomas EJ, Grillo SP. The safety attitudes questionnaire (SAQ): guidelines for administration. Huston: The University of Texas Center of Excellence for Patient Safety Research and Practice, 2003.

8. Zimmerman N, Küng K, Sereika SM, Engberg S, Sexton B, Schwendimann R. Assessing the safety attitudes questionnaire (SAQ), German language version in Swiss university hospitals - a validation study. BMC Health Services Research [Internet] 2013 [access: 12 apr 2016]; 13(347). Available from: http://www.biomedcentral.com/14726963/13/347.

9. Gama ZAS, Oliveira ACS, Hernández PJS. Cultura de seguridad del paciente y factores asociados en una red de hospitales públicos españoles. Cad Saúde Pública [revista en Internet]. 2013 [acceso: 5 oct 2015]; 29(2): 283-293. Disponible en: http://dx.doi.org/10.1590/S0102311X2013000200015. 10. Pereira MD, Souza DF, Ferraz F. Segurança do paciente nas ações de enfermagem hospitalar: uma revisão integrativa da literatura. Rev Inova Saúde [Internet]. 2014 [acesso em: 2 out 2015];3(2):55-87. Disponível em: http://periodicos.unesc.net/index.php/Inova saude/article/viewFile/1746/1672. 
11. Oliveira JLC, Matsuda LM. Vantagens e dificuldades da acreditação hospitalar: A voz dos gestores da qualidade. Esc. Anna Nery [Internet]. 2016 [acesso em: $25 \mathrm{dez}$ 2016]; 20(1): 63-69. Disponível em: http://dx.doi.org/10.5935/14148145.20160009.

12. Barbosa MH, Mendes Sousa E, Felix MMS, Oliveira KF, Barichelo E. Patient safety climate in a hospital specialized in oncology. Rev. Eletr. Enf [Internet]. 2015 [access: 18 jan 2016]; 17(4): 1-9.

Available from:

https://www.fen.ufg.br/revista/v17/n4/pdf/ v17n4a17-en.pdf.

13. Sterud T, Hakon A, Tynes JT. Workrelated psychosocial and mechanical risk factors for low back pain: a 3-year followup study of the general working population in Norway. Occup Environ Med [Internet]. 2013 [acess: 21 jan 2015]; 70:296-

302. Available from: doi: 10.1136/oemed2012-101116.

14. Schwendimann R, Zimmermann N, Küng K, Ausserhofer D, Sexton B. Variation in safety culture dimensions within and between US and Swiss Hospital Units: an exploratory study. BMJ Qual Saf [Internet]. 2013 [acess: 18 aug 2016]; 22(1): 32-41. Available from: doi: 10.1136/bmjqs-2011-000446. 15. Aiken LH, Sermeus W, Heede KV, Sloane DM, Busse R, Mckee M, et al. Patient safety, satisfaction, and quality of hospital care: cross sectional surveys of nurses and patients in 12 countries in Europe and the United States. BMJ Qual Saf. [Internet]. 2012 [acess: $19 \mathrm{dec}$ 2016]; 344: e1717. Available from: http://www.bmj.com/content/344/bmj.e171 7.

16. Fagan MJ. Techniques to Improve Patient Safety in HoZspitals: What Nurse
Administrators Need to Know. J Nurs Adm [Internet]. 2012 [acess: 21 apr 2015]; 42(9): 426-430. Available from: doi: 10.1097/NNA.0000000000000122 17. Patel S, Wu A. Safety Culture in Indian Hospitals: a cultural adaptation of the safety attitudes questionnaire. J Patient Saf. [Internet]. 2014 [access: 19 jan 2016]; 12(2):75-81. Available from:

http://www.ncbi.nlm.nih.gov/pubmed/246 81421.

18. McFadden KL, Stock GN, Gowen CR. Leadership, safety climate, and continuous quality improvement: impact on process quality and patient safety. Health Care Manage Rev [Internet]. 2015 [access: 04 jul 2016]; 40(1): 24-34. Available from: doi: 10.1097/NNA.0000000000000119. 19. Magalhaes AMM, Dall'agnol CM, Marck PB. Nursing workload and patient safety - a mixed method study with an ecological restorative approach. Rev Latino-Am Enferm [Internet]. 2013 [access: 14 oct 2016]; 21(spe):146-54. Available from:

http://dx.doi.org/10.1590/S010411692013000700019.

20. Giehl CT, Costa AEK, Pissaia LF, Moreschi C. A equipe de enfermagem frente ao processo de implantação da Sistematização da Assistência de Enfermagem. Rev Enferm Atenção Saúde [Internet]. 2016 [acess: 10 jan 2017]; 5(2):87-95. Available from: http://seer.uftm.edu.br/revistaeletronica/ind ex.php/enfer.

\section{RECEBIDO: 20/02/2017 \\ APROVADO: 05/07/2017 \\ PUBLICADO: 31/07/2017}

